


\title{
Electric Double Layered Magnetic Fluids (EDL-MF) Based on Spinel Ferrite Nanostructures $\left[\left(\mathbf{M}_{1-x}^{+2} \mathbf{F e}_{x}^{+3}\right)\right]_{A}\left[\left(\mathbf{F e}_{2-x}^{+3} \mathbf{M}_{x}^{+2}\right)\right]_{\mathrm{B}} \mathbf{O}_{4}^{-2}$
}

\author{
F.A. Tourinho \\ Complex Fluid Group, Instituto de Química, Universidade de Brasília \\ J. Depeyrot, G.J. da Silva and M.C.L. Lara \\ Complex Fluid Group, Institute de Física, Universidade de Brasília \\ 70.910 - 900 Brasília - (DF) Brazil
}

Received 29 October, 1998

\begin{abstract}
This paper presents a review of Electric Double Layered Magnetic Fluids (EDL-MF) based on spinel ferrite type $\left[\left(\mathrm{M}_{1-x}^{+2} \mathrm{Fe}_{x}^{+3}\right)\right]_{A}\left[\left(\mathrm{Fe}_{2-x}^{+3} \mathrm{M}_{x}^{+2}\right)\right]_{B} \mathrm{O}_{4}^{-2}$, with $\mathrm{M}(\mathrm{II})=\mathrm{Mn}, \mathrm{Co}, \mathrm{Ni}, \mathrm{Cu}$ and $\mathrm{Zn}$. The chemical synthesis of the nanoparticles using hydrothermal techniques and their complete peptization in an aqueous medium results in a ultra stable magnetic colloid, EDL-MF. The characterization of the particles was performed using X-ray diffraction and electronic microscopy techniques. The resulted sols were investigated by magnetic, magnetooptical measurements and magnetic resonance techniques.
\end{abstract}

\section{Introduction}

MF or ferrofluids are colloidal suspensions of finely divided $(10 \mathrm{~nm})$ magnetic particles dispersed in a specific carrier and constitute a promising technological material. MF where steric repulsion for the particles peptization is used, in polar or non polar medium, are known as surfacted MF; those in water, where the electrostatic principle is used are known as aqueous or ionic MF. Note that in the former case, the term "aqueous" is improperly employed, because it refers to the solvent used, but not to the way used for the particle peptization, as the term "surfacted" means. For instance, we can also have surfacted magnetic fluid in water. In the latter case, these MF are also improperly named "ionic", which can be confused with ionic liquid carriers (melting salts at room temperature), where only ions exist. Thus, we introduce here, for the first time in the magnetic fluid literature, a more realistic term - Electric Double Layered (EDL) Magnetic Fluids - instead of ionic or aqueous MF.

Artificially created many years ago, it was only in the end of the 1980s that the research in MF was introduced in Brazil by Tourinho [1]. An International Advanced School on MF was held on October 3-7 1994 at the Federal University of Goiânia (UFG), Goiânia, Brazil. The course turned out to be a success and many well-known lecturers participated ( see the proceedings appeared in the Brazilian Journal of Physics [2]). At the present moment, it is a growing research theme in some important Brazilian Universities, although, at the same time its potential industrial applications remain difficult to establish.

\section{I.1 General remarks}

Nowadays, homogeneous fluid exhibiting ferromagnetic properties are not found in nature. For instance, iron is magnetizable (ferromagnetic) but in liquid phase (at high temperature), it loses this property, because the Curie Point is over passed and it therefore becomes paramagnetic. On the other hand, mercury is a room temperature liquid metal but it is not magnetizable. The paramagnetic fluids which are aqueous solutions of paramagnetic salts, e.g., $\mathrm{FeCl}_{3}$ or $\mathrm{MnCl}_{2}$ or rare earth 
metal salts exhibit such macroscopic properties only in very intense fields.

In mid-1960s, using new technology, it became possible to combine fluidity properties and the possibility to interact with a magnetic field (earlier considered to be incompatible) in a unique material: a magnetic fluid (ferrofluid). Thus, for the first time in history it became possible to exert a magnetic force on a fluid to control its position. At the same time, the coexistence in such systems of a strong magnetization comparable to that of a solid magnet and fluidity, allowed them to be referred as unique physical objects. Then, the science of MF was created and technological applications have grown rapidly, encouraging theoretical and experimental research in ferrofluid's chemistry, physicochemistry, thermodynamics and mechanics.

\section{I.2 Manufacture of Magnetic Fluids with differ- ent ferrophases and carriers}

MF's are two-phase liquid -a colloid- containing ferro- or ferrimagnetic nanoparticles in a broad spectrum of ordinary non magnetic liquid (carriers). The principle of the stability in such systems is due to the fact that these nanostructured materials, generally denser than the liquid carrier, are animated by the Brownian agitation, thus compensating the force of gravity and being therefore able to be maintained in solution. To avoid their agglomeration, a repulsive character force must be introduced against magnetic dipolar force and Van der Waals interaction and at the same time permits a peptization in non magnetic carrier. Each particle can be considered as a single-domain region and bears a constant magnetic moment which can align or be attracted in the direction of an applied external magnetic field, inducing therefore in such systems a macroscopic magnetic moment. This property is then transmitted to the liquid carrier due to the Brownian motion of the particles thus causing the interaction of the whole fluid, and the field. In conclusion, stable MF can be manufactured using two main principal steps: firstly the production of fine magnetic particles and secondly, their stabilization in a desired carrier liquid.

\section{I.2.1 Particles preparation}

The technological process to produce fine magnetic particles can be mainly divided in two principal categories: dispersion and condensation methods.

\section{Grinding (dispersion) methods}

In the dispersion methods, a sample of magnetic bulk material (magnetite) is broken down into colloidal dimension by a laborious mechanical process.

\section{Condensation methods}

More recently, condensation methods were proposed and involve condensation from a chemical reaction. The condensation methods are faster, cheaper and lead to more versatile products: the nanoscale magnetic particles have a very reactive surface allowing the stabilization of the particles in a large spectrum of media including both polar and non polar solvents. Afterwards, this method has been extended to the synthesis of other types of ferrites in addition to magnetite.

\section{I.2.2 Particles peptization in general carrier medium}

In a second step of the synthesis, the stabilization of the fine ferrite particles is performed by the addition of a barrier to avoid the aggregation which would occur because of Van der Walls and magnetic dipoledipole interactions. The protected particle can be Surfacted (S-MF), Electric Double Layered (EDL-MF) or Biocompatibled (B-MF) and more recently $\mathrm{H}$-bonded (Hb-MF).

\section{Surfacted Magnetic Fluids (S-MF)}

In the ordinary surfacted type, a physical barrier is introduced using a suitable dispersing agent (surfactant). Surfactants are molecules which consist of two well defined regions: one which is oil-soluble (hydrophobic) and other one which is water soluble (hydrophilic). The hydrophobic part is non-polar and usually consists 
in an aliphatic or hydocarbon residue. The hydrophilic part is polar and usually consists in polar groups which can strongly interact with water (specially hydroxyl, carboxyl and ionic groups). So, to stabilize ferrites particles in a non-polar medium, a simple surfactation is required; the hydrophilic part is adsorbed in the particle surface and the hydrophobic part therefore becomes compatible with the solvent. In polar medium, like water, two layers of surfactant are needed to form an exterior hydrophilic layer.

\section{Electric Double Layered (EDL-MF)}

In the EDL-MF a repulsive electrostatic force allows the particles to stay in solution without agglomeration. This can be obtained providing specific adsorption of amphoteric ( $\mathrm{pH}$ dependence) hydroxyl groups which creates an adjustable surface charge density (electric double layer). Therefore, the Electric Double Layered method is more advantageous than the double surfactation method for solubilization in water.

\section{Biocompatibled Magnetic Fluids (B-MF)}

Biocompatible magnetic fluids have to be adapted to biological media (hemocompatibility, toxicity, biodegradability, etc.). Such systems are based on magnetic particles coated with biologically active molecules. In this way, ferrite particles are associated with biological polyelectrolite molecules which brings two or more polyfunctional chemical groups. These ones are responsible for the complex link molecule-particle surface, and for the colloidal stabilization by the creation of an electric double layer. The coated colloidal particle material can be directly introduced in the human body depending on the nature of the particle, and on how this molecules adsorbed onto the particle surfaces work like a bridge to graft on proteins and antibodies.

\section{H-bonded Magnetic Fluids (Hb-MF).}

Recently, Tourinho et al. [3] proposed a new particle petization in polar media without surfactant or electric double layer. This new principle consists of a stabilization by H-bonding between the particle surface and the self-assemblied liquids. It leads to very stable magnetic fluids, principally at high temperatures.

\section{I.3 Applications}

MF can be confined, positioned, shaped and controlled by magnetic forces. Because of this surprising and interesting behaviour MF's are successfully used in sealing applications, vacuum rotary feedthroughs, linear seals, audio loudspeakers and inertia dampers and so on. In the years to come, one of the most important way of development for the magnetic liquids will be the biological domain, principally in drugs vectoring and oncological diagnosis and treatment.

\section{I.4 Historical Aspects}

The first efforts to produce magnetic fluids were done in 1779 by Knight [4], followed by Bitter [5] and Elmore [6] who obtained a suspension of large magnetic particles. Though not fully stable those colloids were similar to the present "magnetic fluids". However, to reach the present synthesis it was necessary to develop both a method to produce stable suspensions and a technique to remove the big particles using through an efficient centrifugation process. The stable magnetic fluids was first obtained by Papell [7], at NASA. They were MF similar to the present commercially available ones and were designed to control the flux of combustible (fuel) in the absence of gravity in outer space. Between 1965 and 1969, Rosensweig [8] managed to produce MF in kerosene and others solvents like water, fluorocarbons and esters. It is interesting to note that until the first quarter of the 70's MF were produced through the suspension of magnetite particles obtained from grinding processes. Then it became necessary to obtain magnetic fluids through faster processes. In 1973 Kalafalla and Reimers [9] first proposed a chemical method for the production of surfacted MF based on magnetite. In 1979, Massart [10] proposed a chemical synthesis where magnetite nanoparticles could be dispersed in polar solvents, without the necessity of surfactants. However, there was a stability problem due to oxidation, i.e., the magnetite transformed into maghemite. It is relevant to observe that this kind of transformation had not happened before because natural magnetite is stable. In 1987, Cabuil [11] forced the oxidation of magnetite in maghemite. In 1989 and 1996, changing the process of the initial synthesis, Tourinho 
$[12,13]$ was able to obtain ultra stable and highly concentrated MF with different magnetic particles based on spinel ferrite type $\left[\left(\mathrm{M}_{1-x}^{+2} \mathrm{Fe}_{x}^{+3}\right)\right]_{A}\left[\left(\mathrm{Fe}_{2-x}^{+3} \mathrm{M}_{x}^{+2}\right)\right]_{B} \mathrm{O}_{4}^{-2}$, with $\mathrm{M}(\mathrm{II})=\mathrm{Mn}, \mathrm{Co}, \mathrm{Ni}, \mathrm{Cu}$ and $\mathrm{Zn}$.

Initially the science of MF was consistent with the idealized physical and mathematical model for the behavior of a single-phase isotropic liquid - a magnetic liquid. At present, MF can not be described by a simplified representation and now the science of MF has become an independent field of investigation, belonging to disciplines as diverse as physicochemistry of colloids, physics of magnetic phenomena, and magnetohydrodynamics. A detailed bibliography dealing with MF is outlined in the Proceedings of the International Conferences on MF [14].

\section{Experimental preparation details of Electric Double Layered Magnetic Fluids based on spinel ferrite type $\left[\left(\mathbf{M}_{1-x}^{+2} \mathbf{F e}+{ }_{x}^{3}\right)\right]_{A}\left[\left(\mathbf{F e}_{2-x}^{+3} \mathbf{M}_{x}^{+2}\right)\right]_{\mathrm{B}} \mathbf{O}_{4}^{-2}$, with $\mathrm{M}(\mathrm{II})=\mathrm{Mn}, \mathrm{Co}, \mathrm{Ni}, \mathrm{Cu}$ and $\mathrm{Zn}$}

\section{II.1 Chemical synthesis of magnetic particles}

The chemical synthesis of charged magnetic nanoparticles based on spinel ferrite type, $\left[\left(\mathrm{M}_{1-x}^{+2} \mathrm{Fe}_{x}^{+3}\right)\right]_{A}\left[\left(\mathrm{Fe}_{2-x}^{+3} \mathrm{M}_{x}^{+2}\right)\right]_{B} \mathrm{O}_{4}^{-2}$, with $\mathrm{M}(\mathrm{II})=\mathrm{Mn}$, $\mathrm{Co}, \mathrm{Ni}, \mathrm{Cu}$ and $\mathrm{Zn}$, dispersed in water allows the production of EDL-MF. Magnetic particles of a ferrofluid must be chemically stable in the liquid carrier and have a convenient size (a few nanometres) to provide colloidal sols. $\mathrm{Mn}, \mathrm{Co}$ [12 ] and $\mathrm{Cu}, \mathrm{Zn}, \mathrm{Ni}$ [13] spinel oxide particles are prepared by condensation method from the chemical reaction among aqueous solutions of metal mixtures in alkaline medium. Particle nature and size are fixed by the coprecipitation step. It is therefore necessary to control all the experimental steps in order to obtain reproducible results. Important parameters to control are the composition of the initial mixtures, the composition of the alkaline medium, the operating mode, the temperature and the ionic strength of the initial mixture. All of them have been tested to succeed in doing such a synthesis. As far as the molar ratio is concerned the best initial condition for all sample prepared corresponds just to the ferrite stoichiometry, i.e., 0.33 . In addition the particular base which is used, the $\mathrm{pH}$ and the temperature are extremely important in the synthesis. The best temperature range for the synthesis is around $100^{\circ} \mathrm{C}$. Usually a base excess is needed due to the acidity of the initial mixture. The reagent addition procedure, including the way and speed of mixing, acts directly to determine the average value of the particle size. The fine particles are obtained by pouring the mixture as quickly as possible into the base medium under vigorous stirring. Very high ionic strength as well as base excess are very important in the synthesis of nickel ferrite particles.

\section{II.2 Peptization of magnetic Particles in aque- ous media - EDL-magnetic fluid preparation}

Finally, after the magnetic particles being synthesized they were washed in acid medium using a ferric nitrate solution at boiling temperature in order to promote the chemical surface stabilization. Very stable and concentrated magnetic fluids are obtained, after the chemical surface stabilization step, by dispersion in acid (alkaline) medium with suitable counterions, like nitrate or perchlorate (tetramethylammonium cation).

Easily available amounts of very concentrated magnetic fluids have already allowed new experiments on the wetting of a platinum wire to be carried out [15]. It has been shown [12] that a large anisotropy constant of cobalt ferrite enables microscopic needle synthesis of this magnetic compound and it is hoped that new applications in the engineering field may arise as a result of this behavior. More recently [16] the birefringence experiments demonstrate the existence of a magnetically ordered core inside the nickel ferrite particles allowing their use in dynamic optical probing for biomedical applications.

\section{Particle Characterization}

\section{III.1 Electronic Microscopy}

MF always present an intrinsic polydispersity [12, 15] and electronic microscopy is generally used to characterize both the geometrical size distribution of the magnetic nanoparticles and their crystalline structure. In the following, we present typical results of electronic microscopy measurements $[16,17]$ performed on a Transmission Electronic Microscope JEOL 100CX2 after dispersion of the dilute fluid sample by ultrasonic 
waves. Picture 1 shows a direct image of nickel ferrite magnetic particles which appear roughly spherical. Considering one thousand and a half particles (not involved in aggregates) it leads to the size histogram displayed in Fig. 1. For all magnetic fluids samples and whatever the way of preparation of fine particles, the size distribution of magnetic particles is, in a first approximation, usually well described by a log-normal distribution of diameters $\mathrm{P}(\mathrm{d})$ :

$$
P(d)=\frac{1}{\sqrt{2 \pi} d \sigma} \exp \left[-\frac{\ln ^{2}\left(d / d_{0}\right)}{2 \sigma^{2}}\right]
$$

where $\mathrm{d}_{0}$ corresponds to the mean value of $\mathrm{d}$ and $\sigma$ is the standard deviation. In Fig. 1, we also show a best fit (solid line) of the size histogram which corresponds to $\mathrm{d}_{0}=3.5 \mathrm{~nm}$ and $\sigma=0.15$. However one can note that the fitted distribution does not account for the tail of the histogram which represents the biggest particles. Moreover, due to the experimental process which requires the solvent evaporation, the number of small aggregates is rather underestimated. It is therefore important to remind that such a picture is not a direct image of the real fluid.

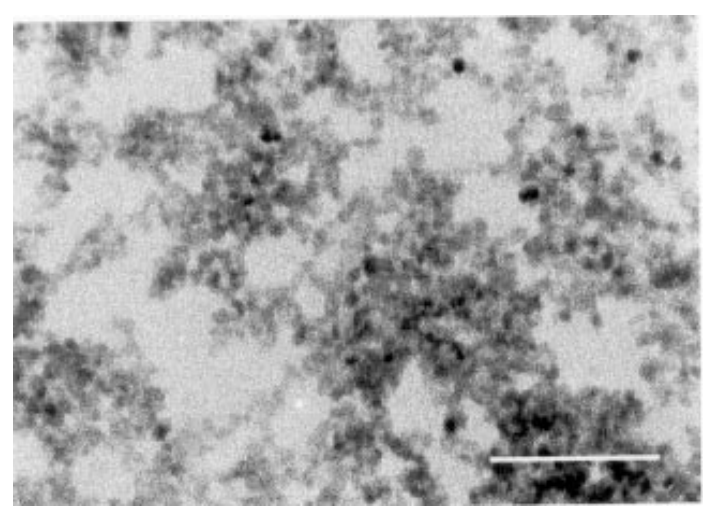

Picture 1. Electronic microscopy picture of a chemically synthesized EDL-MF sample made of nickel ferrite particles. The scale represents $50 \mathrm{~nm}$.

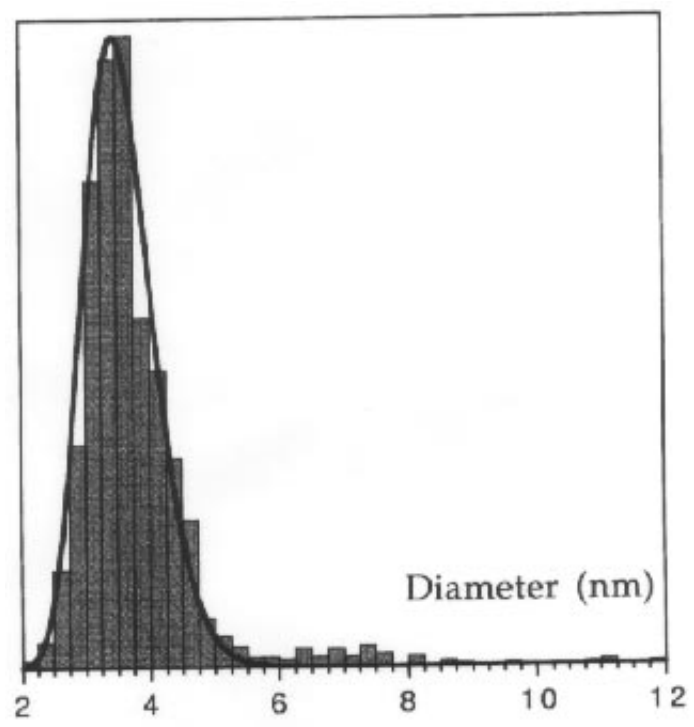

Figure 1. Size histogram of the Nickel EDL-MF sample deduced from electronic microscopy images (one thousand and a half particles). A best fit of this histogram by a log-normal distribution law gives a mean diameter $d_{0}=3.4 \mathrm{~nm}$ and a standard deviation $\sigma=0.15$.

The electronic microscopy also gives the opportunity to perform a crystalline characterization. The diffraction of the electron beam by ferrite nanoparticles leads to a typical diffraction pattern as it is displayed in Picture 2 in the case of Nickel ones. The indexation of the observed lines can be deduced from a comparison to the diffraction pattern of a gold sample. Their positions and relative intensities allows us to identify the structure of these magnetic particles which is of spinel-type [18].

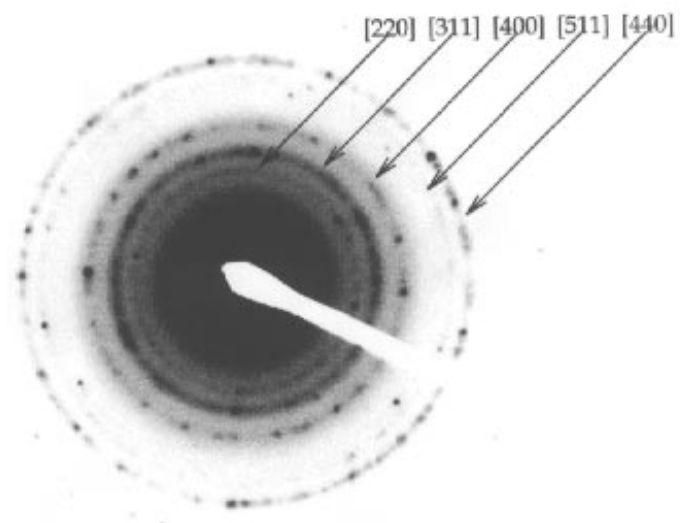

Picture 2. Electron diffraction pattern of nickel ferrite EDLMF sample. The indexation of the observed lines corresponds to the characteristic interplanar spacings of the spinel structure. 


\section{III.2 X-ray Diffraction}

As the particles size plays a fundamental role in both the magnetic and magneto-optical behaviour, it remains essential to perform a carefull particle characterization. Investigations of the nanoparticles by X-rays diffraction [19] constitute an additional method in order to confirm the crystalline structure. Focusing on the line broadening, it will also provide another determination of the particles size. In this way, only the crystalline part of particles is taken into account. Xrays diffraction measurements are generally realized on a powder obtained by evaporation of the liquid carrier of the sample. We present in Fig. 2 typical powder difratograms performed on three magnetic fluid samples based on $\mathrm{CuFe}_{2} \mathrm{O}_{4}, \mathrm{ZnFe}_{2} \mathrm{O}_{4}$ and $\mathrm{NiFe}_{2} \mathrm{O}_{4}$ ferrites, using a Rigaku/Geigerflex X-rays diffractometer and the $\mathrm{Cu}-\mathrm{Ka}$ radiation at $\mathrm{l}=1.54 \AA$. All of them exhibit several lines corresponding to the characteristic interplanar spacings [220], [311], [400], [511] and [440] of the spinel structure. The broadening of the peaks which arises from the finite dimension of the crystal (polycristalline specimens) is essentially due to the roughly spherical particle diameter, acording to the Debye-Scherrer equation:

$$
d_{R X}=\frac{K \lambda}{\Delta \cos \theta}
$$

where $\mathrm{K}=0.9$ is a correction factor to account for particle shapes, $\theta$ is the Bragg angle and $\Delta$ is the observed breadth at half the maximum peak intensity. One has to mentionned that the observed width includes additional sources of broadening, arising from the experimental set up and instrumention, which were, in our measurements, determined using a Si standard monocrystal. Then, the particle sizes are generally determined by calculating the mean diameters associated with the most intense X-ray peak [16, 17, 19], corresponding to the [311] one, and gives in our case the results shown in Table I. As the X-rays diffraction provides a mean diameter one has to note that this experimental technique favours the biggest particles. Therefore, X-rays diffraction determination of the particles diameter generally gives a higher size when compared to electronic microscopy results.
Table I: Mean particle diameter estimated from the width at half maximum of the [311] diffraction peak for EDL-MF samples based on $\mathrm{CuFe}_{2} \mathrm{O}_{4}, \mathrm{ZnFe}_{2} \mathrm{O}_{4}$ and $\mathrm{NiFe}_{2} \mathrm{O}_{4}$ in powder form.

\begin{tabular}{cc}
\hline Magnetic nanoparticles & $\begin{array}{c}\text { X-ray diffraction mean } \\
\text { diameter } d_{0 . R X}(\mathrm{~nm})\end{array}$ \\
\hline $\mathrm{CuFe}_{2} \mathrm{O}_{4}$ & 8.5 \\
$\mathrm{ZnFe}_{2} \mathrm{O}_{4}$ & 6.3 \\
$\mathrm{NiFe}_{2} \mathrm{O}_{4}$ & 4.4 \\
\hline
\end{tabular}

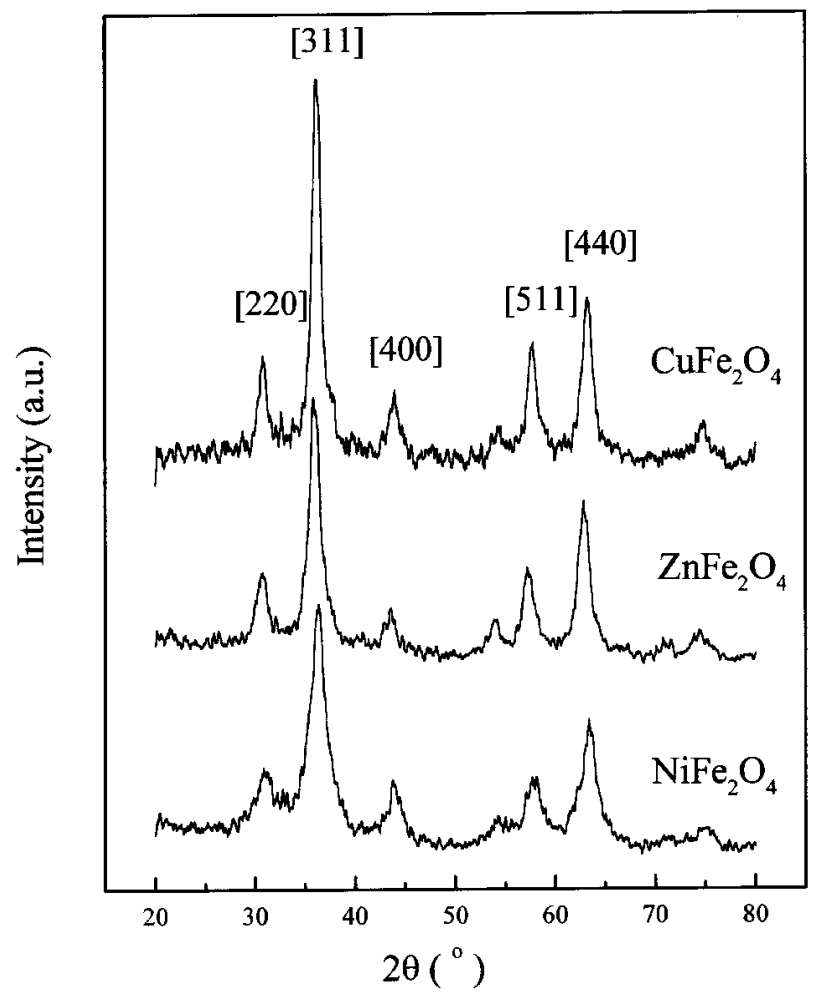

Figure 2. X-rays powder diffractogram of Copper, Zinc and Nickel ferrite nanoparticles. Characteristic lines [220], [311], [400], [511] and [440] of the spinel structure are indexed.

\section{Magnetic Properties}

\section{IV.1 Theoretical background}

The magnetic properties of magnetic fluids arise from the dispersion of magnetic nanoparticles [20, 21] in a liquid carrier, typically $10^{16}$ part. $\mathrm{cm}^{-3}$ in dilute solution. The combination of the properties of the crystalline spinel structure and of the exchange interactions 
between ions of the tetraedric sites and ions of the octaedric ones leads to a global ferrimagnetic order in the core grain [22]. Moreover, as the diameter particle is ranging from 2 to $15 \mathrm{~nm}$, it is always smaller than the thickness of a Bloch wall. Each magnetic grain can therefore be considered as a single magnetic monodomain which bears a magnetic moment $\mu$ which is of the order, for ferrites particles, of $10^{4} \mu_{B}, \mu_{B}$ being the Bohr's magneton. At the equilibrium, this magnetic moment is held oriented in the direction of the easy axis of magnetization by an energy barrier of anisotropy $\mathrm{E}_{a}$. At zero magnetic field, the rotation of the magneticmoment is a thermally activated process, characterized by the Néel relaxation time $\tau_{N}=\tau_{0} \exp \left(-\mathrm{E}_{a} / \mathrm{k}_{B} \mathrm{~T}\right)$, where $\tau_{0}$ is the spin relaxation time which is about $10^{-9} \mathrm{~s}, \mathrm{k}_{B}$ is the Boltzman constant and $\mathrm{T}$ the temperature.

In the liquid solution, freedom degrees of mechanic rotation allow a second relaxation process, the Brownian rotation characterized by a Brownian relaxation time $\tau_{B}=3 \eta \mathrm{V}_{h} / \mathrm{k}_{B} \mathrm{~T}$, where $\eta$ is the fluid viscosity at the temperature $\mathrm{T}$ and $\mathrm{V}_{h}$ the hydrodynamic particle volume. In aqueous samples this time is typically of about $10^{-6} \mathrm{~s}$.

In a dilute regime where interparticle interactions are negligible, MF therefore present a giant paramagnetic behaviour, the so-called superparamagnetism [23, $34,25]$. In the absence of magnetic field, the orientation of the particles magnetic moment is randomly distributed, and the resulting magnetization is equal to zero. As the magnetic field increases, the permanent moments are free to align along the field and their orientation distribution results from a balance between the magnetic energy of the particles and their thermal one. At the thermal equilibrium, the Boltzmann distribution can be applied [26, 27] and the resulting orientation distribution of magnetic moments can be written:

$$
\langle\vec{\mu}\rangle=L_{1}(\xi)=\operatorname{coth} \xi-1 / \xi
$$

where $\xi=\mu \mathrm{H} / \mathrm{k}_{B} T$ is the Langevin parameter and $\mathrm{L}_{1}(\xi)$ is the first Langevin law. In high external magnetic field, all the magnetic moments are aligned in the field direction and the magnetization saturates. The magnetization does not present any hysteresis.

However, the simple description based on the Langevin formalism has to be modified taking into ac- count the magnetic fluids polydispersity and the normalized magnetization becomes:

$$
\frac{M(H)}{m_{S} \phi}=\frac{\int_{0}^{\infty} d^{3} L_{1}[\xi(d)] P(d) d d}{\int_{0}^{\infty} d^{3} P(d) d d}
$$

where the saturation value of the magnetization is $\mathrm{M}_{\text {sat }}=\mathrm{m}_{s} \phi, \mathrm{m}_{s}$ being the saturation magnetization of the magnetic grain and $\phi$ the volumic fraction of magnetic material in the solution.

Such a model applies rather well for EDL-MF based on $\gamma-\mathrm{Fe}_{2} \mathrm{O}_{3}$ particles, at room temperature [25], assuming for the nanomaterial, a magnetization of the order of $75 \%$ of the bulk one for particle diameter about $10 \mathrm{~nm}$.

\section{IV.2 Magnetization measurements}

The curve $\mathrm{M}(\mathrm{H}) /$ Msat therefore reduces to a function of two parameters $\mathrm{d}_{0}$ and $\sigma$ if $\mathrm{m}_{s}$ is known [25]. Then, by fitting magnetization experimental measurements one can obtain another determination of mean magnetic sizes of the particles and standard deviations of the size distribution.

We present in Figs. 3 and 4 some experimental room temperature measurements performed with a Foner's device on dilute $(\phi<0.8 \%)$ EDL-MF based on $\mathrm{MnFe}_{2} \mathrm{O}_{4}$ [12] and $\mathrm{NiFe}_{2} \mathrm{O}_{4}$ [17] particles respectively. The ferrofluid solutions magnetization is equal to zero in zero field, does not present any hysteresis in the investigated range of field and is an increasing function of the applied field. All these results as well as those of ref. [25] confirm the existence of an ordered magnetic core which, under an applied magnetic field, induces a mechanical rotation of the particles, dragged by the rotation of the core magnetic moment.

Moreover, as in the case of niquel ferrites particles, the magnetization is proportional to the volume fraction $\phi$ of nanomaterial, (see on Figs. 4 and 5) a result which shows that, at least from a magnetic point of view, no interparticles interactions has to be taken into account. In the case of Mn-based samples, one can easily obtain the parameters of the size distribution which are $\mathrm{d}_{0}=5.9 \mathrm{~nm}$ and $\sigma=0.45[12]$. 


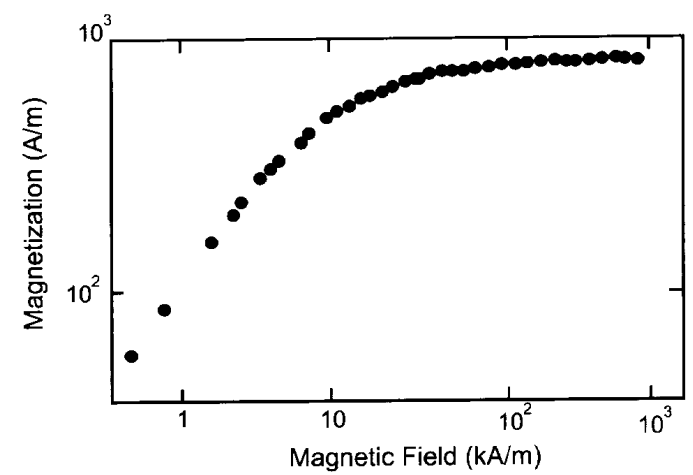

Figure 3. Magnetization $M$ of an EDL-MF based on manganese ferrite nanoparticles in a log-log representation. A best fit using the formalism (4) gives $d_{0}=5.9 \mathrm{~nm}$ and $\sigma=$ 0.45 .

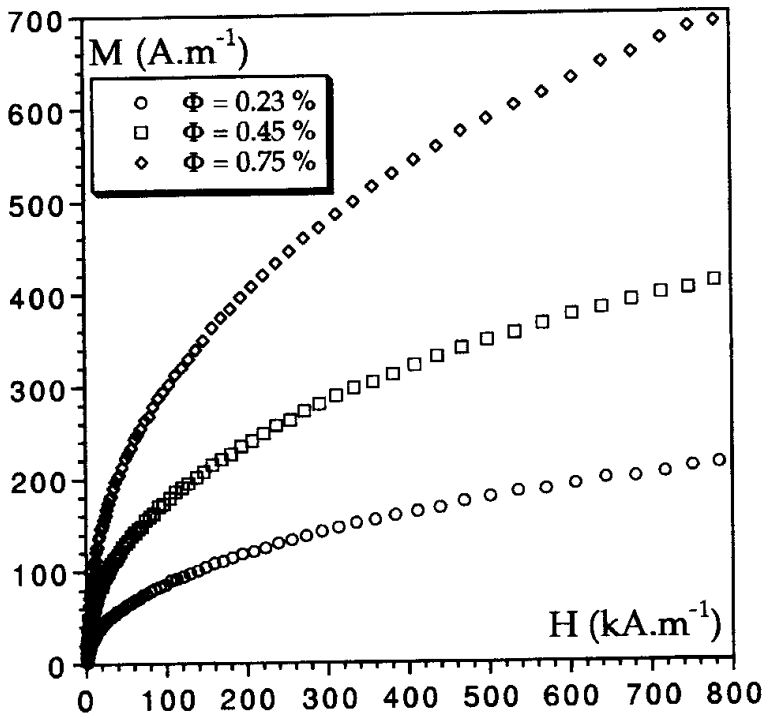

Figure 4. Magnetization M of EDL-MF's based on nickel ferrite nanoparticles (three samples at three volume fraction $\Phi)$ ) as a function of the applied magnetic field $H$ in a linear representation.

Besides experiments performed on liquid solutions, where Brownian rotation is usually the dominant process which tends to align the magnetic moment of particles along the applied field direction, measurements in frozen solutions can provide informations about the nanomaterials magnetic anisotropy. When ferrofluid nanoparticles are frozen in a tight polymeric matrix, the Brownian rotation is inhibited and the mechanism of the moment rotation is due to thermal fluctuations of the magnetic moment within the magnetic particle. Such measurements have been performed at room temperature on EDL-MF based on $\mathrm{CoFe}_{2} \mathrm{O}_{4}$ and $\mathrm{MnFe}_{2} \mathrm{O}_{4}$ [12] particles and show that Mn-based samples do not exhibit any remanence while Co-based samples exhibit a large one. These results are in good agreement with the calculated ratio between the anistropy energy and the thermal one and the comparison between the bulk anistropy constants of these two materials, which is aproximately fifty time larger for $\mathrm{CoFe}_{2} \mathrm{O}_{4}$ than for $\mathrm{MnFe}_{2} \mathrm{O}_{4}$, allow to explain the magnetic behaviour of those frozen solutions.

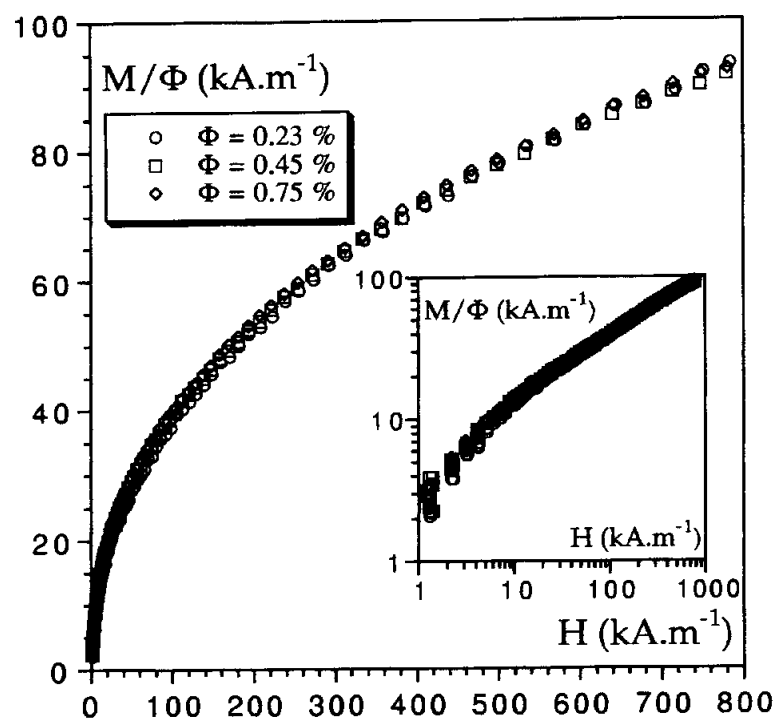

Figure 5. Magnetization $M / \Phi$ as a function of the applied magnetic field $H$ in a linear representation for the three samples. The inset displays the same results in a log-log representation.

In the case of Ni-based samples, figure 4 and 5 clearly show that in the investigated range of magnetic fields the magnetization does not saturate. Such a result has very recently $[17,28]$ been explained by the existence of a strong surface magnetism which would find its origin in the small size of the particles and in the nickel ferrite crystalline structure. One has to remind that the existence of a spin disorder all around an external layer of the magnetic particles has also been proposed in maghemite particles [29, 30]. A carefull analysis of the Ni-based samples magnetization shows one standard contribution coming from the magnetic core which follows a Langevin formalism and another one coming from a large number of surface spins badly correlated to the ferrimagnetic core structure. This analysis gives a mean diameter and a standard deviation equal to $4.2 \mathrm{~nm}$ and 0.55 respectively. Standard deviations deduced from magnetization measurements are generally greater that those provided by electronic 
microscopy since all the small agregates in solution contribute to the magnetic measurement [12].

\section{Magneto-optical behaviour}

\section{V.1 Theoretical background}

Chemically synthesized EDL-MF based on $\gamma-\mathrm{Fe}_{2} \mathrm{O}_{3}$, $\mathrm{CoFe}_{2} \mathrm{O}_{4}$ and $\mathrm{NiFe}_{2} \mathrm{O}_{4}$ particles are optically active and exhibit strong optical birefringence under magnetic fields. Isotropic in zero field, the magnetic fluid solutions become optically uniaxial under an applied field [23, 31]. Such a macroscopic effect saturates in high fields and is related to the microscopic optical anisotropy of the particles and to their progressive orientation along the field direction. A field induced aggregation of particles is totally rejected by Small Angle Neutron Scattering (SANS) experiments [32] if the ionic strength of the solutions is less than 0.05 mol. $^{-1}$ with particles of superficial density of charges of the order of $0.2 \mathrm{C} . \mathrm{m}^{-2}$. The origin of the optical anisotropy of those nanoparticles had been highly discussed and the birefringence may have several origin:

- a field-induced effect in the particle material [33], however, within the accuracy and in the field range of standard measurements, no optical anisotropy of the solutions is observed if the particles are dispersed in a zero magnetic field in a carrier subsequently frozen (for example, a tight polymeric matrix).

- the internal optical anisotropy of the magnetic particles, but as tested by X-rays diffraction measurements the ferrite crystalline structure of the particles is cubic: it is incompatible with the generation of birefringence. A tetragonal order of vacancies has been put in evidence in bulk $\gamma-\mathrm{Fe}_{2} \mathrm{O}_{3}$ [34] by the observation of additional superstructure reflections. However it has been shown [35] that this effect depends on crystallite size and the superstructure reflections disappear for mean particles size smaller than $20 \mathrm{~nm}$; it therefore can not be expected in ferrofluid nanoparticles.

- a shape anisotropy of the particles, but as showed on electronic microscopy pictures, the particles are more rock-like shaped than elongated.

Recently, the optical anisotropy [36] as well as the magnetic anisotropy [30, 37], of chemically synthesized $\gamma-\mathrm{Fe}_{2} \mathrm{O}_{3}$ particles have been attributed to a surface contribution related to a possible disordered layer of spins at the surface of the nanoparticles.
The results of static experiments performed on dilute solutions may be interpreted in terms of a Langevin formalism [36, 38, 39] coupled with a log-normal size distribution of particles and taking into account an optical anisotropy of individual particles $\delta \mathrm{n}_{0}$ :

$$
\frac{\Delta n}{\delta n_{0} \phi}=\frac{\int_{0}^{\infty} L_{2}(\xi) d^{3} P(d) d d}{\int_{0}^{\infty} d^{3} P(d)}
$$

where $\mathrm{L}_{2}(\xi)=1-(3 / \xi) \mathrm{L}_{1}(\xi)$ is the second Langevin law.

\section{V.2 Birefringence measurements}

We present in the following two optical probing of magnetic fluids based on nickel ferrite particles, performed in the presence of a static magnetic field (see on Fig. 6) and of a low alternating one (see on Fig. 7). Static field experiments were already performed on $\mathrm{Co}-$ and $\gamma-\mathrm{Fe}_{2} \mathrm{O}_{3}$ - based samples and the used experimental setup as well as the obtained results were published elsewhere [38, 39].

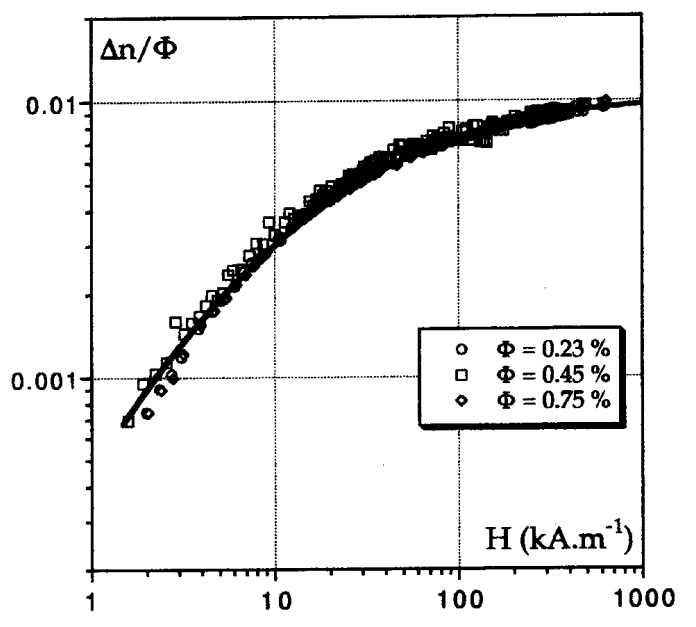

Figure 6. Log-log representation of $\Delta n / \Phi=f(H)$ for nickel EDL-MF's samples. The full line is a best fit using (5) which gives $d_{0}=6.4 \mathrm{~nm}$ and $\sigma=0.55$. 


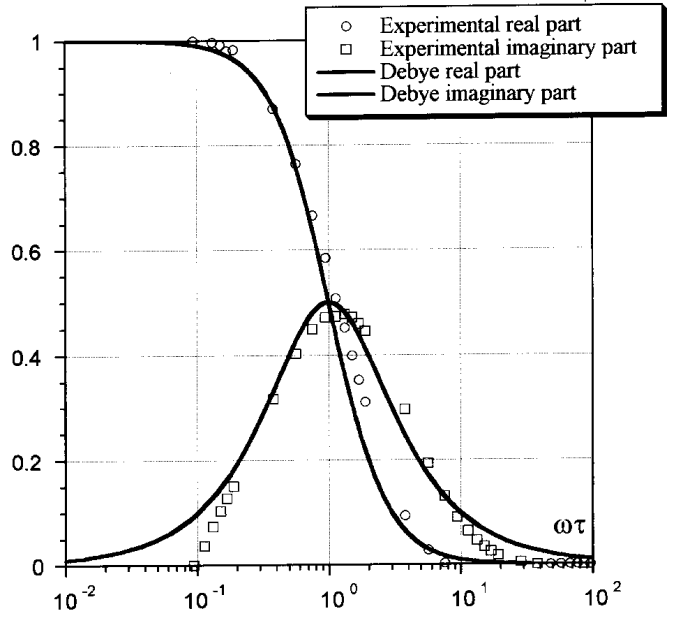

Figure 7. Real and imaginary parts of the birefringence of the nickel EDL-MF sample as a function of frequency. The full lines correspond to the real and the imaginary parts of the Debye relaxation.

\section{Static birefringence $[17,28]$}

The static birefringence is given in figure 6 , for three $\mathrm{Ni}$-based samples of different volumic fraction $\phi$, in a reduced representation as a function of the applied static magnetic field $\mathrm{H}$. As for the magnetization curves, one can note that $\Delta n$ is equal to zero in zero applied field, no hysteresis is observed in the experimental range of magnetic field and $\Delta n$ is proportional to the volume fraction $\phi$. The previous formalism correctly fits the experiments with a quite large size distribution of particles. Assuming in a first approximation the magnetization of the particles equal to that of the bulk, it allows to determine a characteristic optical distribution of diameters $d_{0}=6.4 \mathrm{~nm}$ and a standard deviation $\sigma=0.55$. These values, as those one determined from magnetic measurements, indicate that such samples are rather polydisperse. It should be noted that static birefringence is particularly sensitive to eventual aggregates and, on the contrary, totally insensitive to small superparamagnetic particles not aligned by the magnetic field.

\section{Dynamic birefringence [28]}

A dynamic probing is also performed in a birefringence measurement, under a low alternating magnetic field, as a function of the field frequency. The nickel ferrofluid sample, dispersed in glycerine, is submitted to an alternating field $=16 \mathrm{kAm}^{-1}$. and the field frequency $\mathrm{f}$ is experimentally ranging from $1 \mathrm{~Hz}$ to $1 \mathrm{kHz}$ [16].

As a function of frequency, we present in Fig. 7 two parameters respectively proportional to the real and to the imaginary part of the birefringence normalized at $1 \mathrm{~Hz}$ : . The imaginary part presents a maximum at $\mathrm{f}=15 \mathrm{~Hz}$. We compare in Fig. 7 the experimental results to a standard Debye relaxation:

$$
\Delta n / \Delta n(1 H z)=1 /(1+i \varpi \tau)
$$

Such a model expresses that at low frequencies the particle optical axis follows the oscillations of the magnetic field. As the frequency increases the phase-lag between the field and the particle axis progressively increases and in the high frequency limit the particle does not oscillate anymore. Identifying the frequency of the maximum of the imaginary part to the condition $\varpi \tau=1$ allows a comparison of the model to the experiment as a function of the reduced parameter $\varpi \tau$. The characteristic time resulting from this adjustment is $\tau=10 \mathrm{~ms}$. This Debye relaxation time corresponds to a Brownian time of rotational diffusion. We find here $d_{h}=30 \mathrm{~nm}$ to compare with the low field average of static birefringence $[16,36] d_{L F}=20 \mathrm{~nm}$ and with a transient measurement after a pulse of magnetic field in an aqueous sample made of the same particles: $\tau=$ $27 \mu \mathrm{s}$ and $d_{h}($ pulse $)=40 \mathrm{~nm}$. These large values of $d_{h}$ have to be correlated to the large width of the particle distribution in the sample and to the low field value (16 $\mathrm{kA} \cdot \mathrm{m}^{-1}$ ) used in the experiment.

\section{Magnetic Resonance Studies}

The electronic paramagnetic resonance technique (EPR) is very efficient for studying complex systems like for instance MF, mainly when the interactions considered are particle-particle and particle-solvent. From the analyses of the EPR spectra one can obtain information on the constant of anisotropy for grains, the effect of the ionic force on ionic magnetic fluids, changes on the effective radius of the floating particles, Brownian 
relaxation time and particles concentration as a function of temperature. Facts like change on the line width with temperature, resonance field position, broadening of the line width as a function of particle-particle distance are some aspects of the technique which allow us to understand better the magnetic fluid system.

The EPR technique was first used by Sharma and Walder [40] on 1977 to study S-MF. From 1993 many studies has been made on synthetic MF fluids by Tourinho [41], as for example, EDL-MF based on $\left(\mathrm{MnFe}_{2}\right.$ $\mathrm{O}_{4}$ ), where a set of samples at various concentration were synthesized. Particle-particle interactions on these samples has been studied through the analyses of the linewidth variation with temperature from the EPR spectra. From these studies it was possible to estimate the interaction energy among the particles[42], clarifying some aspects on the magnetic interactions.

To analyze the variation of the line width with temperature, for different concentrations, it was used a model based on the Van Vleck momenta method $[42,43]$. On Fig. 8 it is shown the experimental data of the magnetic fluid $\mathrm{MnFe}_{2} \mathrm{O}_{4}$ in different concentrations the variation of the line width with temperature based on the model ref. $[42,43]$.

It was also, studied the characterization of this magnetic fluid through the particle-particle interaction by comparing two systems. The single particle system and the magnetic agglomerates. Where one could observe a quenching on the linewidth broadening which is associated to a new relaxation channel [44]. Fig. 9 shows magnetic fluids (single particles) and agglomerates.

Mean while the model referred in ref. [44], could not explain the linewidth variation with temperature for ferrofluid samples with magnetic particles floating on querosene [45]. The fitting of the linewidth variation with temperature for these samples, did not follow the model applied on EDL-MF based on manganese ferrite particles.

\section{VI.1 The magnetic resonance characterization of highly anisotropic magnetic fluids using doping procedure}

As a matter of fact the transition metal ions play an important role on the studies of complex systems through the paramagnetic resonance technique. They are usually utilized as a structural probe to study var- ious substances. The general procedure consist usually to dope a sample with a well-known paramagnetic ion and to attribute to the changes of the resonance parameters as due the environment of these ions [46].

In terms of the MF all the magnetic resonance data published to date of the reference [53], presents the magnetic nanoparticles themselves as the resonant centers. In this case the effective magnetic field at the particle site is a combination of the external field, the anisotropy field and the fluctuation field [47]. As a consequence, as far as the microwave frequency is concerned, highly anisotropic MF may require very low excitation frequencies [48]. Indeed, for highly anisotropic MF, magnetic resonance at a fixed frequency may not represented a promising characterization technique as it did for moderated anisotropic MF.

To overcome the difficulties mentioned above, there was a proposition of to dope a highly anisotropic MF with a well known paramagnetic center (for example with $\mathrm{Cu}^{2+}, \mathrm{Mn}^{2+}$, etc.) and magnetic resonance was now focused on the dopant center instead of the magnetic particle itself. The attention was concentrated on the resonance lineshape of the dopant center instead of the magnetic particle itself. It was attributed to the presence of the magnetic particles of the fluid the resulting difference of the lineshape before and after the doping procedure. To describe the dopant paramagnetic ion resonance lineshape in doped magnetic fluids it starts averaging out a lorentzian or a gaussian shaped resonance line to the angular dependence of the anisotropy field of the surrounding magnetic particles. This is done by considering the effective field at the dopant site as a combination of the external field and the parallel component of the anisotropy field. Meaning parallel component of the anisotropy field the component of the dipolar field due to the surrounding magnetic particles parallel to the external magnetic field, here taken as the z-direction. The angular average of the dipolar field over the resonance lineshape is performed by integrating the effective field from $\theta=0$ through $\theta=\pi$, being $\theta$ the angle with respect to the z-direction. In a second step It is performed a radial average of the parallel component of the dipolar field to the resonance lineshape by integrating from a radial distance close to the particle surface $\left(\mathrm{r}_{1}\right)$ to a radial distance greater than the particle radius $\left(\mathrm{r}_{2}\right)$. In a third 
and last step It was necessary take into account the particle size dispersity considering a log-normal distribution of the particle radius and we average out the particle radius from zero to infinity. Indeed the resonance lineshape of the dopant ion in the doped MF is described by

$$
L=A \int_{0}^{\infty} P\left(R, R_{0}, \sigma\right) d R \int_{r_{1}}^{r_{2}} r^{2} f(r, T) d r \int_{0}^{\pi} g\left(H, H_{d}\left(r, \theta, R, I_{s}\right), H_{0}, \gamma\right) d \theta
$$

where $A$ is the dopant ion molar concentration in the doped MF, $\mathrm{H}$ is the external sweeping field, $\mathrm{P}\left(\mathrm{R}, \mathrm{R}_{0}, \sigma\right)$ is the log-normal distribution function, eq. 1 .

$g\left(H, H_{d}\left(r, \theta ; R, I_{s}\right), H_{0}, \gamma\right)$ is the first derivative of a lorentzian or a gaussian function, depending which of them best fit the dopant itself. $H_{0}$ and $\gamma$ are the resonance field and the linewidth at half height of the dopant resonance line. The average value of the particle radius and the broadening of the particle size distribution are described by the parameters $\mathrm{R}_{o}$ and $\sigma$, respectively. $\mathrm{H}_{d}=$ $(4 \pi / 3) M_{s} \cos (\theta+\phi)\left(3 \cos ^{2} \theta+1\right)^{1 / 2} \sin \theta(\mathrm{R} / \mathrm{r})^{3}$ is the component of the dipolar field parallel to the external field $H$, where $\phi=\tan ^{-1}(1 / 2 \tan \theta)$.

The function $f(r, T)$ is the normalized Boltzmann factor to describe the place where the dopant ions are distributed among the particles under the action of the potential of the double layers from each particle.

$$
f(r, T)=\frac{\exp \left(-2 e \Psi(r) / k_{B} T\right)}{\int_{r_{1}}^{r_{2}} \exp \left(-2 e \Psi(r) R / k_{B} T\right) d r}
$$

where $e$ is the electron charge, $k_{B}$ is the Boltzmann constant, $T$ the temperature and $\Psi$ is the electrostatic potential seeing by the dopant ion due to double layer around each magnetic particle.

$\Psi$ obtained from the solution of the PoissonBoltzmann $\nabla^{2} \Psi=-4 \pi \rho / \varepsilon$, where $\rho$ is the diffuse ions concentration around a colloidal particle, given by, $\rho=\sum_{i} n_{i} z_{i} \exp \left(-z_{i} e \Psi / k_{B} T\right) n_{i}$ is the number concentration of the ionic species of valence $z_{i}$. The boundary conditions to Poisson-Boltzmann are

$$
\Psi_{\text {interface }}=\Psi_{0}
$$

$$
(\nabla \Psi) . n_{\text {surface }}=-4 \pi \sigma_{s} / \varepsilon
$$

where $\Psi_{0}$ is the potential at surface of the colloidal particle charged with electrical superficial density of charge $\sigma_{s}$ and $n$ is the unitary vector normal to this surface.

For spherical particles there is analytical solution only for some situations where the potential $\Psi$ is low, meaning Debye-Huckel approximation. The majority solutions are numerical or some mixing of analytical and numerical [49-52]. One important parameter is the Debye length, defined as $\mathrm{k}^{-1}=\left\{\left(4 \pi e^{2} / \varepsilon k_{B} T\right) \sum_{i} n_{i}^{0} z_{i}^{2}\right\}^{-1 / 2}$ It gives the extent of the electric double layer surrounding each particle.

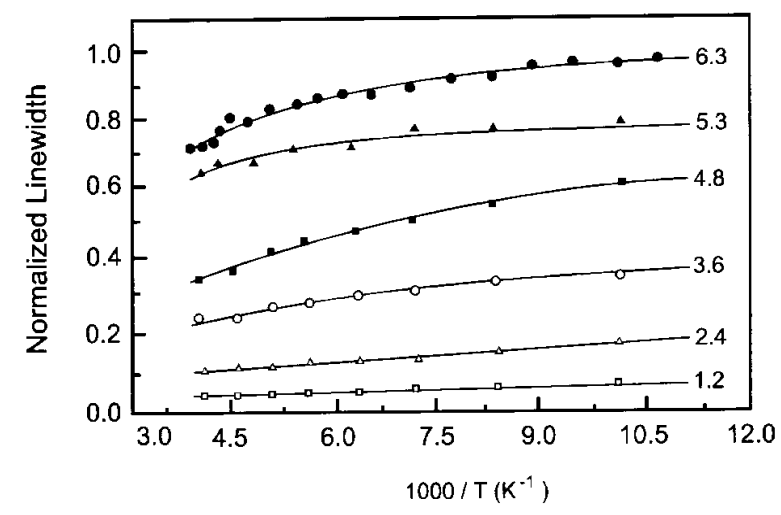

Figure 8. Normalized magnetic - resonance linewidth broadening is plotted as a function of $1000 / \mathrm{T}\left(\mathrm{K}^{-1}\right)$. Particle concentration related to each samples is indicated in units of $10^{16}$ particles $/ \mathrm{cm}^{3}$. The linewidth data were normalized with respect to the sample containing $6.3 \times 10^{16}$ particles $/ \mathrm{cm}^{3}$. 


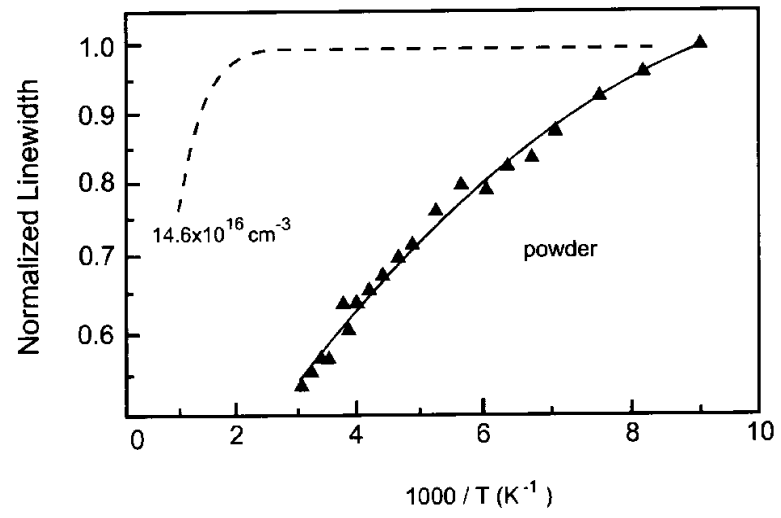

Figure 9. Normalized magnetic - resonance linewidth broadening is plotted as a function of $1000 / \mathrm{T}\left(\mathrm{K}^{-1}\right)$ for the powder sample (solid triangles) obtained from the starting magnetic fluid sample. The continuous line through the solid triangles represents the best fit. The dashed curve represents the expected resonance -linewidth broadening for a magnetic fluid sample containing $14.6 \times 10^{16}$ particles $/ \mathrm{cm}^{3}$.

\section{Application of the model}

This is model was applied to analyze a EDLMF cobalt-ferrite $\left(\mathrm{CoFe}_{2} \mathrm{O}_{4}\right)$ water-based $[53,54]$ doped with $\mathrm{Cu}^{2+}$ ion in a wide range of the dopant concentration with a strong evidence that the magnetic fluid stability (no phase separation) was preserved despite of the increasing ionic strength due to $\mathrm{Cu}^{2+}$ ion addition.

At a first step, with this procedure, the particle size polydispersity parameters, meaning $\mathrm{R}_{o}$ and $\sigma$, of the cobalt-ferrite water-based magnetic fluid was obtained. There was an excellent agreement between the parameters $\mathrm{R}_{o}$ and $\sigma$ obtained from this procedure and that from the electron microscopy results $\left(\mathrm{R}_{o}=7.8 \mathrm{~nm}\right.$ and $\sigma=0.23$ ), which are considered the standard technique used for that purpose. The full lines in Fig. 10 represent the best fit of the experimental resonance lines according to Eq. 1 for various concentration of the dopant. Table II shows the $\mathrm{R}_{o}$ and $\sigma$ values for each concentration of the dopant ion.

Table II: Adjusted values for $R_{0}$ and $\sigma$ as a functions of the $\mathrm{Cu}^{2+}$ dopant molar concentration.

\begin{tabular}{ccc}
\hline $\mathrm{Cu}^{2+}$ ion $(\mathrm{mol} / \mathrm{l})$ & $R_{0}(\mathrm{~nm})$ & $\sigma$ \\
\hline 0.025 & 7.80 & 0.22 \\
0.050 & 7.79 & 0.22 \\
0.075 & 7.80 & 0.23 \\
0.100 & 7.81 & 0.23 \\
0.150 & 7.81 & 0.23 \\
\hline
\end{tabular}

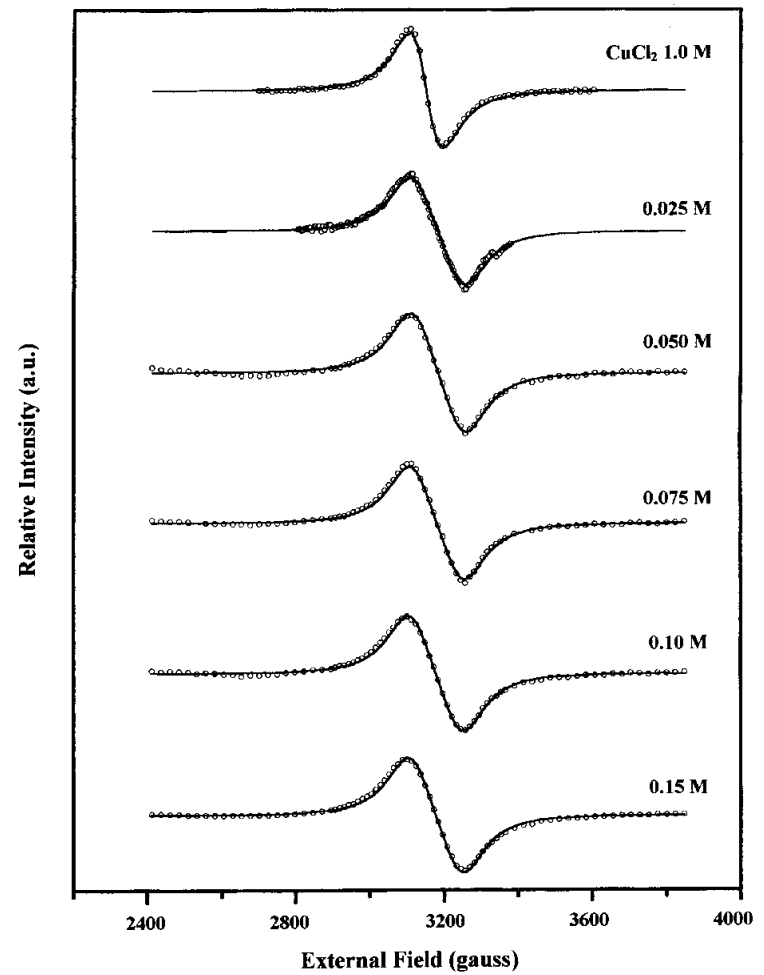

Figure 10. The open circles are the experimental data obtained from an EPR spectrometer at X-Band, model ESP $300 \mathrm{E}$ made by Bruker. - The full lines are the fitting to theoretical models: for $\mathrm{CuCl} 2$ at the concentration of $1.0 \mathrm{M}$ (molar) a lorentzian fits well, and the other points are fitted to equation 1 for a solution $\mathrm{o} \mathrm{CuCl}_{2}$ diluted at the ratio 1 part to 3 , in the ferrofluid sample. The values of these concentration are listed near each curve.

According to the scheme proposed in that work and considering the "GLS" analytical approximation model for higher potentials[52] It was possible to estimate the Debye length of the electrical double layer for this sample, performing a radial average of the parallel component of the dipolar field to the resonance lineshape by integrating from a radial distance close to the particle surface $\left(\mathrm{r}_{1}=R+1 / k\right)$, where $\mathrm{R}$ is the radius of each particle and $1 / k$ is the Debye length to a radial distance $\left(r_{2}\right)$ corresponding to the electric double layer of the neighbor particle. The result was in excellent accordance with was known for that sample. That work ends pointing to the following important conclusion: the sample preparation is much simpler and the time of measurement much shorter than in the case of both electron microscopy and magnetization.

\section{Concluding remarks}

Since the first preparation (and application) of MF in the 60 's, there has been an enormous progress that 
shows a clear account for the magnetic fluid fundamental physical understanding. However the synthesis of very stable $\mathrm{MF}$ is still a refinement of empirical methods. In conclusion, we are reviewing in this paper the synthesis of electric double layered (EDL) MF based on spinel ferrite type $\left[\left(\mathrm{M}_{1-x}^{+2} \mathrm{Fe}_{x}^{+3}\right)\right]_{A}\left[\left(\mathrm{Fe}_{2-x}^{+3} \mathrm{M}_{x}^{+2}\right)\right]_{B} \mathrm{O}_{4}^{-2}$, with $\mathrm{M}(\mathrm{II})=\mathrm{Fe}, \mathrm{Mn}, \mathrm{Co}, \mathrm{Ni}, \mathrm{Cu}$ and $\mathrm{Zn}$. The fine control of parameters like temperature, ionic strength, initial molar fraction, nature of the reaction medium are very important as far as the success of the preparation is concerned. We found that the close control of these parameters determines the average particle size, magnetic nature of the particles and surface reactivity. X- ray diffractograms and electron microscopy are presented as the first characterization techniques. The polidispersity is then obtained as described above and the mean particle diameters provided by both techniques are in good agreement.The study of the magnetic and magneto-optical behavior does not only provide another determination of the size distribution but both magnetization and birefringence measurements also allow the study of surface effects as well as relaxation processes. Moreover,experiments in crossed alternating and static magnetic fields would give important informations about the magnetic anisotropy.

Magnetic resonance technique has been proved to be powerful on the characterization of EDL-MF with additional advantage that the sample preparation is much simpler and the time of measurements much shorter than in the case of both electron microscopy and magnetization. Instead several other physical techniques, the sample amount used in magnetic resonance is minimum.

\section{Acknowledgements}

Authors are indebted to Prof. R. Perzynski (UPMC), Dr. E. Hasmonay (UPMC), and Prof. R. Itri (USP) for very helpful discussions. We wish to thank to CNPq, CAPES, FAP-DF, PADCT for financial support.

\section{References}

1 F. A. Tourinho, Ferrofluides à Base de Ferrite de Cobalt et Ferrite de Manganese, Elaboration, Comportement Physicochimique et Magnétique. Thèse de Doctorat D'État ès Sciences Physiques, Université Pierre et Marie Curie, Paris, France (1988).
2 Proceedings from First South American Advanced School on Magnetic Fluids, Brazilian Journal of Physics, 25 (1995).

3 F. A. Tourinho and M. H. Sousa, 1998. Private Communication, University of Brasília, Brazil.

4 See E. P. Wohlfarth, Ferromagnetic Materials, Vol. 2, pp. 512, North-Holland Publishing Co., Amsterdam, 1982.

5 F. Bitter, Phys. Rev. 41, 507 (1932).

6 W. C. Elmore, Phys. Rev. 54, 309 (1938).

7 S. S. Papell, US Patent 3215572, 1965.

8 See E. P. Wohlfarth, Ferromagnetic Materials Vol. 2, pp. 518, North-Holland Publishing Co., Amsterdam, 1982.

9 S. E. Khalafalla and G. W. Reimers, US Patent 3764540, 1973.

10 R. Massart, French Patent 7918842, 1979.

11 V. Cabuil and R. Massart, J. Chem. Phys. 84, 967 (1987).

12 F. A. Tourinho, R. Franck, R. Massart and R. Perzynski, Prog. Colloid Polym. Sci. 79, 128 (1989). F. A. Tourinho, R. Franck and R. Massart, J. of Mat. Sci. 25, 3249 (1990).

13 F. A. Tourinho, P. C. Morais, M. H. Sousa and L. G. Macedo Proc. 3nd Internat. Conf. on Intelligent Materials, edited by P. F. Gobin and J. Tatibouet (The International Society for Optical Engineering, Bellingham,Washington-USA) pp. 317 (1996).

14 Proceedings from VI International Conference on Magnetic Fluids, J. Magn. Magn. Mater., 122 (1993); Proceedings from VII International Conference on Magnetic Fluids, J. Magn. Magn. Mater., 149 (1995); Proceedings from VIII International Conference on Magnetic Fluids, J. Magn. Magn. Mater., to be published (1998).

15 J-C Bacri, R. Perzynski, D. Salin and F. A.Tourinho, Europhys. Letters 5, 547 (1988).

16 E. Hasmonay, J. Depeyrot, M. H. Sousa, F. A. Tourinho, J. C. Bacri and R. Perzynski, J. Magn. Magn. Mater. (to appear in 1998).

17 E. Hasmonay, J. Depeyrot, M. H. Sousa. F. A. Tourinho, J. C. Bacri, R. Perzynski and Yu. L. Raikher, submitted for publication (1998).

18 ASTM, n 39.1346

19 L. Q. Amaral and F. A. Tourinho, Brazilian Journal of Physics 25, 142 (1995).

20 Magnetic Properties of Fine Particles, J. L. Dormann and D. Fiorani Eds., North Holland, Amsterdam (1992).

21 J. L. Dormann, E. Tronc and D. Fiorani, in Advances in Chemical Physics Series, vol. 98, 283, John Wiley \& Sons, New-York (1997).

22 L. Néel, C. R. Acad. Sci. 228, 664 (1949). 
23 Magnetic Fluids and Applications Handbook, B. Berkovski Ed., Begell House Inc. Publ., New-York (1996).

24 R. W. Chantrell, J. Popplewell and S. W. Charles, Physica 86, 1421 (1977).

25 J.-C. Bacri, R. Perzynski, D. Salin, V. Cabuil and R. Massart, J. Mag. Mag. Mat. 62, 36 (1986).

26 Yu. L. Raikher and M. Shliomis, Adv. Chem. Phys. 87, 595 (1994).

27 Magnetic fluids, E. Blums, A. Cebers and M. M. Maiorov, Walter de Gruyter, Berlin (1997).

28 E. Hasmonay, Thesis, Université Pierre et Marie Curie, Paris 6 (1998).

29 F. Gazeau, E. Dubois, M. Hennion, R. Perzynski and Yu. L. Raikher, Europhys. Lett. 40, 575 (1997).

30 F. Gazeau, J.-C. Bacri, F. Gendron, R. Perzynski, Yu. L. Raikher, V. I. Stepanov and E. Dubois, J. Mag. Mag. Mat. (to appear in 1998).

31 J J.F. Saenger, K. Skeff Neto, P. C. Morais, M. H. Sousa and F-A. Tourinho, J. Magn. Reson. 133 (1998).

32 E. Dubois, Thesis, Université Pierre et Marie Curie, Paris 6 (1997).

33 J. Ferre and G. A. Gehring, Report on Progress in Physics 47, 513 (1984).

34 C. Greaves, J. Sol. State Chem. 49, 325 (1983).

35 K. Haneda and A. H. Morrish, Sol. State Com. 22, 779 (1977).

36 E. Hasmonay, E. Dubois, J.-C. Bacri, R. Perzynski, Yu. L. Raikher and V. I. Stepanov, The European Physical Journal B (to appear in 1998).

37 V. Shilov, F. Gazeau, J.-C. Bacri, F. Gendron, R. Perzynski and Yu. L. Raikher (to be published).

38 J.-C. Bacri, V. Cabuil, R. Massart, R. Perzynski and D. Salin, J. Mag. Mag. Mat. 65, 285 (1987).
39 S. Neveu-Prin, F. A. Tourinho, J.-C. Bacri and R. Perzynski, Colloids Surf. A80, 1 (1993).

40 V. K. Sharma, T. Walder, J. Appl. Physics, 49, 10 (1977).

41 A. L. Tronconi,, P. C. Morais, F. Pelegrini, and F. A. Tourinho, J. Magn. Magn., Mater. 122, 90 (1993).

42 P. C. Morais, M. C. F. L. Lara, A. L. Tronconi, F. A. Tourinho, F. Pelegrini and A. R. Pereira, J. Appl. Phys. 79 (10), 7931 (1996).

43 J. H. Van Vleck, Phys. Rev. 74, 1668 (1948).

44 P. C. Morais , M. C. F. L. Lara and F. A. Tourinho, J. Magn. Reson., 121, 70 (1996).

45 I. Hrianca, I. Malaescu, F. Calici, C. N. Marin, Abstracts - Eight International Conference on Magnetic Fluids, Romania (1998).

46 M. C. F. L. Lara, Thesis, USP, São Carlos, Brazil (1993).

47 G. J. da Silva, Thesis, UFMG, Belo Horizonte, Brazil (1997).

48 A. M. Konn, P. Laurent, P. Talbolt e M. Le Floch, J. Magn. Magn. Mat., 140-144, 367 (1995).

49 A. L. Loeb, J. Th.G. Overbeek e P. H. Wiersema, The Electric Double Layer Around a Spherical Colloidal Particle, MIT Press, Cambridge, Massachutes., (1961).

50 N. E. Hoskin e S. Levine, Phil. Trans. Roy. Soc. A, 248, 433 (1956).

51 T. H. Gronwall, V. K. La Mer, K. Sandved, Z. Physik, 29, 358 (1928).

52 L. R. White, J. Chem. Soc. Faraday II, 73, 577 (1977).

53 G. J. Da Silva, P. C. Morais e F. A. Tourinho, J. Phys. Chem., 100, 14269 (1996).

54 G. J. Da Silva, P. C. Morais e F. A. Tourinho, J. Chem. Phys., 107, 1 (1997). 